\title{
Investigation of Complex Networks of Spin-Spin Coupling by Two-Dimensional NMR
}

\author{
AD BaX* AND RAY FreEMAN \\ Physical Chemistry Laboratory, Oxford University, Oxford $O X 13 Q Z$, England
}

Received April 9, 1981

\begin{abstract}
Proton-proton couplings in a sample of 9-hydroxytricyclodecan-2,5-dione were studied by the two-dimensional Fourier transform method proposed by Jeener. Various techniques were tested for reshaping the two-dimensional responses to remove the long dispersion-mode tails, and to emphasize cross-peaks at the expense of diagonal peaks. Operation with a mixing pulse of relatively small flip angle allows relative signs of coupling constants to be determined by inspection of the two-dimensional spectrum. Weak long-range couplings, normally hidden within the linewidth, may be detected, although their magnitudes can only be estimated very approximately. A simple modification of the pulse sequence permits broadband decoupling in one frequency dimension, giving proton spectra without any $J$ splittings.
\end{abstract}

\section{INTRODUCTION}

The very first two-dimensional Fourier transform NMR experiment was carried out by Jeener ( 1 ) and later analyzed in detail by Aue et al. (2). The concept was soon realized to be very general, and was extended to two-dimensional $J$ spectroscopy $(3-5)$, the correlation of proton and carbon-13 chemical shifts $(6-8)$, investigations of chemical exchange $(9-11)$, and the indirect detection of multiplequantum transitions $(12-15)$. These extensions were so successful that they diverted attention away from the original experiment in the form described by Jeener $(l)$, where a simple sequence of two $90^{\circ}$ pulses was applied to a proton spin system. One possible factor contributing to this neglect is that the experiment cannot be described in terms of a simple physical picture based on precessing magnetization vectors, but requires a density matrix treatment, which is quite cumbersome for systems of several coupled spins.

Nevertheless, even without a rigorous analysis, this experiment can be very useful for studying the pattern of proton-proton couplings in molecules of intermediate complexity $(16-18)$. The present work investigates various methods of simplifying the resulting two-dimensional spectrum so that spin couplings are more readily assigned, and suggests modifications which render the technique more sensitive to the effects of long-range coupling. It is demonstrated that one such modification permits the relative signs of homonuclear spin-spin coupling constants to be obtained by inspection. Another variation generates a proton spectrum

* Present address: Department of Applied Physics, Delft University of Technology, Delft, The Netherlands.

0022-2364/81/090542-20\$02.00/0 
from which all $J$ splittings have been removed (provided that the coupling is first order). This "broadband decoupling" of protons compares favorably with the established method, in which a $45^{\circ}$ projection of a two-dimensional $J$ spectrum is calculated (3). It is interesting to note the analogy between two-dimensional Fourier transform NMR and several different types of double irradiation experiment.

\section{DESCRIPTION OF THE EXPERIMENT}

In its simple form Jeener's experiment employs the radiofrequency pulse se:quence

$$
\left(T_{\mathrm{w}}-90^{\circ}-t_{1}-90^{\circ}-t_{2}-\right)_{n}
$$

to a system of homonuclear coupled spins, usually protons. The spectrometer receiver is activated only during the detection period $t_{2}$, acquiring a series of signals $S\left(t_{2}\right)$ as the sequence is repeated $n$ times with the evolution period $t_{1}$ incremented in small steps $\Delta t_{1}$ over a suitable time range $n \Delta t_{1}$ of the order of the spin-spin relaxation time $T_{2}$. Between each sequence a period $T_{w}$ is allowed for spin-lattice relaxation. Information about the evolution of the nuclear spin magnetization vectors during $t_{1}$ is coded into the modulation of the detected signals $S\left(t_{2}\right)$ and a data matrix $S\left(t_{1}, t_{2}\right)$ is built up and stored on a disk.

Two-dimensional Fourier transformation of $S\left(t_{1}, t_{2}\right)$ generates a two-dimensional spectrum $S\left(F_{1}, F_{2}\right)$ which contains three distinct categories of signal (2). Any nuclear magnetization that was aligned along the $Z$ axis just before the second $90^{\circ}$ pulse (the "mixing" pulse) gives rise to a series of lines along the $F_{2}$ axis essentially identical with the conventional proton spectrum. These "axial peaks" are of little interest for the purpose of studying spin-spin coupling, so it is convenient to suppress them by alternating the phase of the second radiofrequency pulse (18).

Two other types of signal appear in the two-dimensional spectrum (Fig. 1). The first are peaks which fall on or near the principal diagonal $\left(F_{1}=F_{2}\right)$; these are the resonances grouped around the coordinates $\left(\delta_{\mathrm{A}}, \delta_{\mathrm{A}}\right)$ or around $\left(\delta_{\mathrm{B}}, \delta_{\mathrm{B}}\right)$ of Fig. 1 . They arise from magnetization which either maintains the same resonance frequency during $t_{1}$ and $t_{2}$ (these peaks fall exactly on the diagonal) or is transferred to another resonance in the same spin multiplet ("parallel" transitions). When the mixing pulse is $90^{\circ}$ these peaks all have the same phase; the two-dimensional dispersion; that is to say, sections through such a response parallel to the $F_{1}$ or $F_{2}$ axes have a pure dispersion-mode profile.

It is the third type of signal on which attention is focused. These "cross-peaks" lie at a distance from the principal diagonal at coordinates determined by the chemical shifts of two coupled nuclei, and thus serve to identify coupled resonances. Cross-peaks arise from magnetization that was precessing in the transverse plane during $t_{1}$ and which is transferred to a second nuclear spin by the mixing pulse, thus changing its precession frequency by an amount comparable with the chemical shift difference $\delta_{\mathrm{A}}-\delta_{\mathrm{B}}$ between the two coupled sites. Thus in first-order spectra, cross-peaks lie so much further from the principal diagonal than any diagonal peaks that they are readily distinguished. Cross-peaks are naturally grouped into "'two-dimensional spin multiplets,' in the simple case illustrated in Fig. 1 a square pattern centered at $\left(\delta_{\mathrm{A}}, \delta_{\mathrm{B}}\right)$ or at $\left(\delta_{\mathrm{B}}, \delta_{\mathrm{A}}\right)$, with a splitting $J$ in both dimensions. 


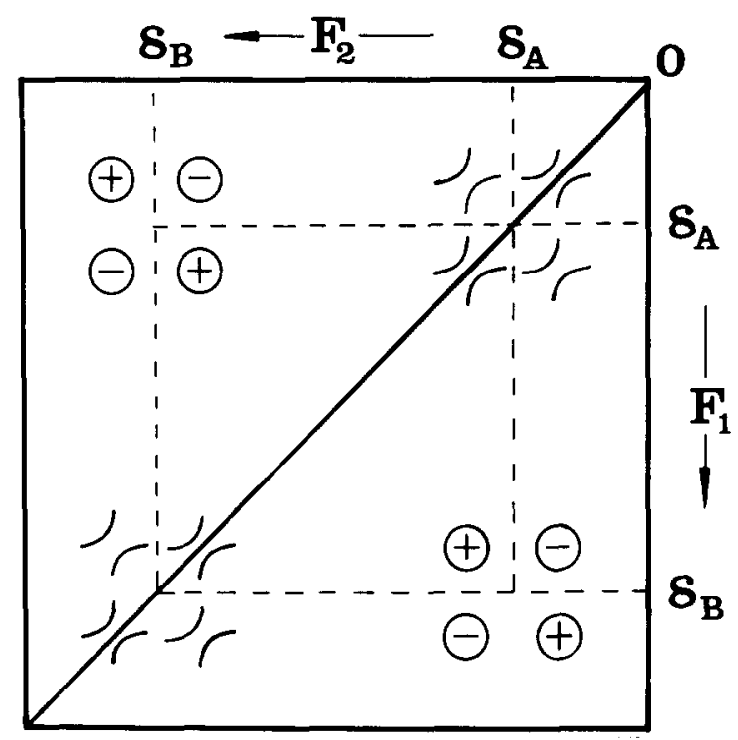

FIG. 1. Schematic diagram of the Jeener spectrum of an AB spin system, showing the diagonal peaks which have a dispersion shape in both dimensions, and the cross-peaks (circles) which have an absorption shape in both dimensions but which alternate in sense $(+,-)$. Diagonal peaks are centered at the coordinates $\left(\delta_{\mathrm{A}}, \delta_{\mathrm{A}}\right)$ and $\left(\delta_{\mathrm{B}}, \delta_{\mathrm{B}}\right)$ while cross-peaks are centered at $\left(\delta_{\mathrm{A}}, \delta_{\mathrm{B}}\right)$ and $\left(\delta_{\mathrm{B}}, \delta_{\mathrm{A}}\right)$. Crosspeaks indicate spin coupling between $A$ and $B$.

Each individual component is a two-dimensional absorption, and the intensities alternate as indicated in Fig. 1. This has the important consequence that if the multiplet components are incompletely resolved, mutual cancellation occurs, and in the limit the cross-peaks vanish. It is therefore essential to ensure that the digitization of the multiplet is sufficiently fine in both dimensions that mutual cancellation is avoided. When a wide range of chemical shifts has to be covered, this entails a large data matrix, which is one of the principal limitations on the method. The antiphase nature of the multiplet components may be put to good use in order to emphasize the cross-peaks in comparison with diagonal peaks. In the time domain, the vectors which give rise to a cross-peak always start out in antiphase and only give a strong resultant if their time development is permitted for a suitably long period. Consequently time-domain weighting functions which increase with time and reach a maximum after an interval of the order of $(2 J)^{-1}$ favor cross-peaks at the expense of diagonal peaks, and shaping functions designed to achieve this are described in detail below. Another consequence of the antiphase nature is that when the absolute-value mode is employed, the dispersion-mode tails of cross-peaks tend to cancel, whereas the tails of diagonal peaks are much more pronounced because the dispersion contributions reinforce.

\section{PRACTICAL ILLUSTRATION}

Many of the modifications and refinements of the Jeener experiment can only be illustrated by reference to actual two-dimensional spectra. These were obtained 


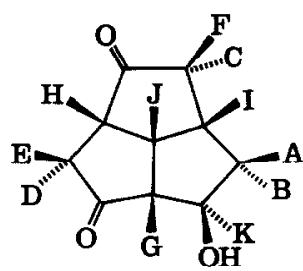

Fig. 2. The compound used for most of the two-dimensional Fourier transform experiments: one of the stereoisomers of 9-hydroxytricyclodecan-2,5-dione.

mainly from the compound sketched in Fig. 2, a tricyclodecane derivative chosen because of the wealth of proton-proton couplings that can be detected. Spectra were recorded on a Varian XL-200 spectrometer with software modifications for two-dimensional Fourier transformation. The phase of the second radiofrequency pulse (the " mixing" pulse) was cycled in $90^{\circ}$ steps as described earlier (18) in order to discriminate the signs of the $F_{1}$ frequencies and thus reduce the size of the data matrix, and also to suppress the unwanted axial peaks. More sophisticated modifications of this pulse sequence are described in the relevant sections below.

\section{Lineshapes}

Two-dimensional spectra commonly use the absolute-value mode of presentation. This has the unfortunate result that the two-dimensional lineshape has marked ridges running through the center of the response parallel to the $F_{1}$ and $F_{2}$ axes; that is to say, the intensity contours form a four-pointed star rather than an ellipse $(19,20)$. This effect has been discussed at some length for a two-dimensional Lorentzian response, but it is less well known that the two-dimensional Gaussian also has ridges if it is recorded in the absolute-value mode.

For the reasons discussed above, it is the diagonal peaks which have the most prominent tails, whereas cross-peaks are much narrower at the base, as can be appreciated from Fig. 3. Interference between the ridges of two different diagonal peaks can give rise to a spurious peak in the two-dimensional spectrum which might be mistaken for a true cross-peak. There is an example of this effect at the bottom left of Fig. 3. The ridges from strong diagonal peaks may make it difficult to pick out cross-peaks that lie near the principal diagonal. It is therefore important to eliminate dispersion-mode components from the two-dimensional lineshape, generating responses with circular intensity contours. This can be achieved by multiplying the time-domain signals $S\left(t_{1}\right)$ and $S\left(t_{2}\right)$ by one of the shaping functions described below.

A second reason for reshaping the time-domain signals is that this is a powerful method of enhancing the intensities of cross-peaks relative to the diagonal peaks. Because the components of a spin multiplet making up a cross-peak are in antiphase and can cause mutual cancellation, a resolution enhancement function increases the intensity of cross-peaks relative to diagonal peaks. Viewed in the time domain, the multiplet components may be represented as vectors which start out in antiphase and require a time of the order to $(2 J)^{-1}$ to precess relative to one 


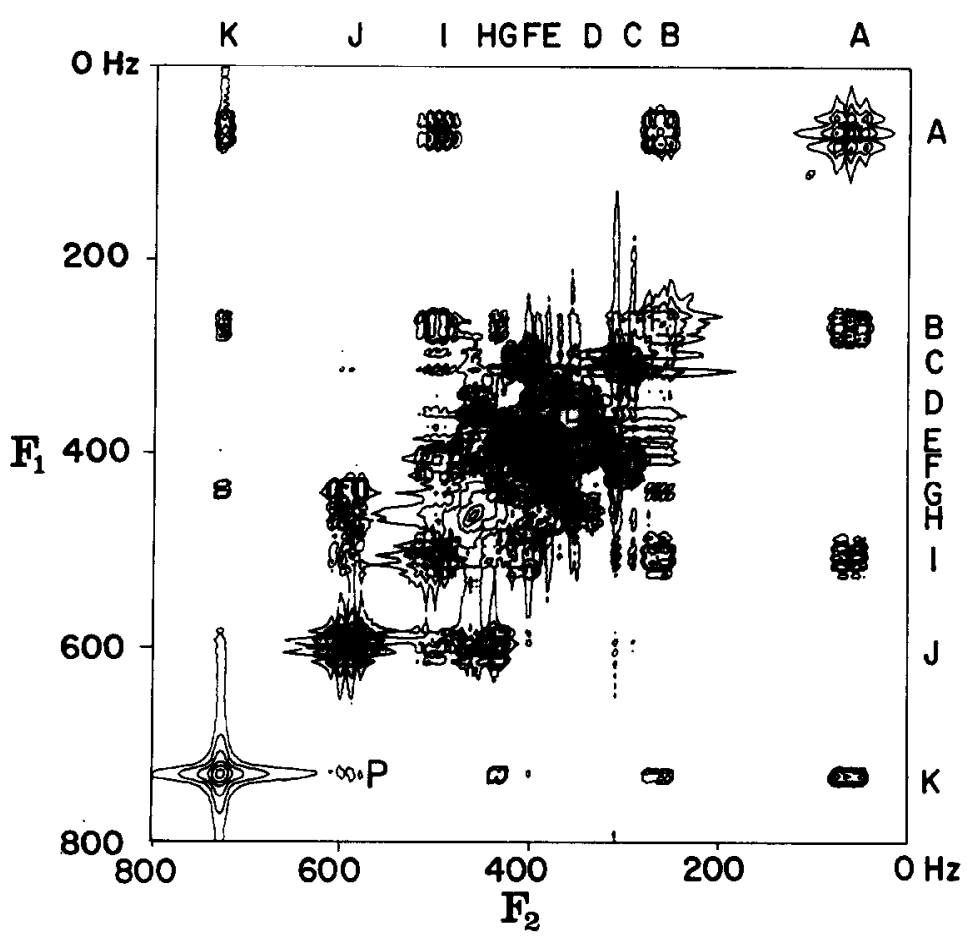

Fig. 3. Jeener spectrum of the tricyclodecane derivative shown as an intensity contour plot. The time-domain signals have been subjected to a shaping function which transforms an exponential decay into a Gaussian decay, giving Gaussian profiles in the frequency domain. Since an absolutevalue mode has been recorded, the diagonal peaks show long tails in the $F_{1}$ and $F_{2}$ dimensions, but the cross-peaks, having equal numbers of antiphase multiplet components, do not show these tails. At the bottom left, marked $P$, is a spurious peak generated by the overlap of the tails of two diagonal peaks.

another to induce a detectable signal. A third reason for imposing a resolution enhancement function on the time-domain signals is that it can be approximately matched to the shape of the coherence transfer echo, described in more detail below.

One resolution enhancement procedure which has been used successfully is convolution difference (2l) employing a multiplying function of the form

$$
f(t)=1-\exp \left(-t / T_{\mathrm{C}}\right), \quad \text { where } \quad T_{\mathrm{C}} \simeq 1 /(2 J) .
$$

This method was applied in both time dimensions to produce the multiple-trace plot of the Jeener spectrum of the tricyclodecane derivative (Fig. 4). Note the absence of ridges in this absolute-value-mode spectrum. Convolution difference first broadens the lines (causing mutual cancellation of antiphase signals) and then subtracts the unbroadened line. In this way the cross-peaks appear at almost full intensity while the diagonal peaks, where such cancellation cannot occur, are reduced in intensity.

There is another shaping function that may be used which has been specifically designed to eliminate dispersion-mode components altogether. It does so by 


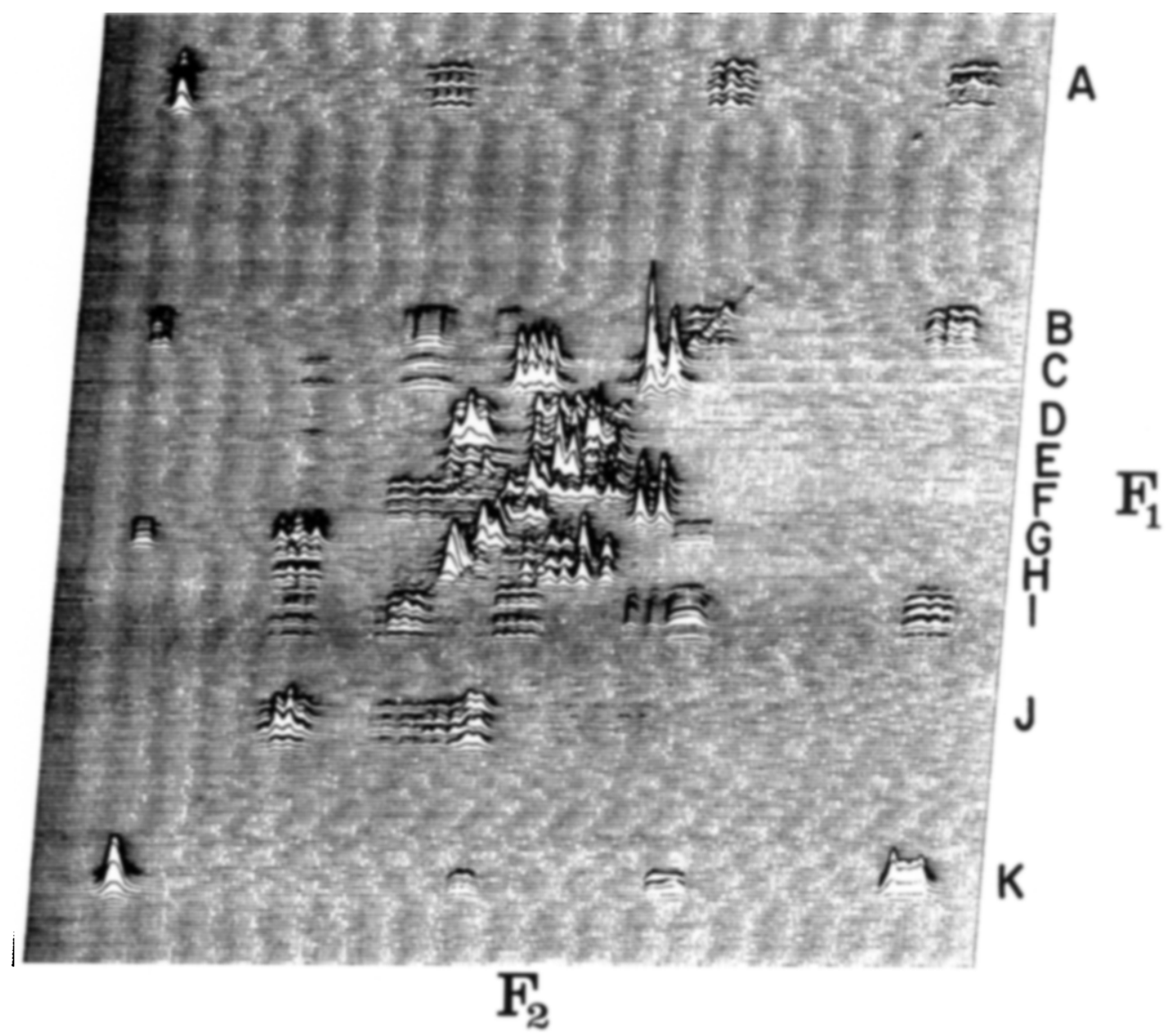

FIG. 4. The multiple-trace plot corresponding to the contour plot of Fig. 3. A convolution-difference resolution-enhancement function has been used in both dimensions in order to reduce the intensity of the diagonal peaks in relation to the cross-peaks and to reduce the tails on the diagonal peaks. The absolute-value mode has been used.

imposing exact symmetry on the envelope of the time-domain signal about its midpoint $t=T / 2$, where $T$ is the acquisition period. The resulting signal has been called a " "pseudoecho," since it has the property of a true spin echo (when $T_{2} \gg T_{2}^{*}$ ) that the dispersion-mode contributions in each half are equal and opposite in sign (22). Fourier transformation of a whole echo (23) or pseudoecho therefore produces a frequency-domain signal which is in the pure absorption mode. However, the pseudoecho differs from the true echo in that the various frequency components are not in phase at the center $t=T / 2$ but at $t=0$. If $\phi$ is the phase of a typical component of the pseudoecho at $t=T / 2$, then the sine and cosine transforms contain that component in the proportion $\sin \phi$ and $\cos \phi$. A straightforward calculation of the sine transform would therefore show gross intensity anomalies; however, the square root of the sum of the squares of the sine and cosine transforms can be recorded, and this contains only the pure absorption mode. Any shaping function can be used which gives the pseudoecho a symmetrical envelope. 


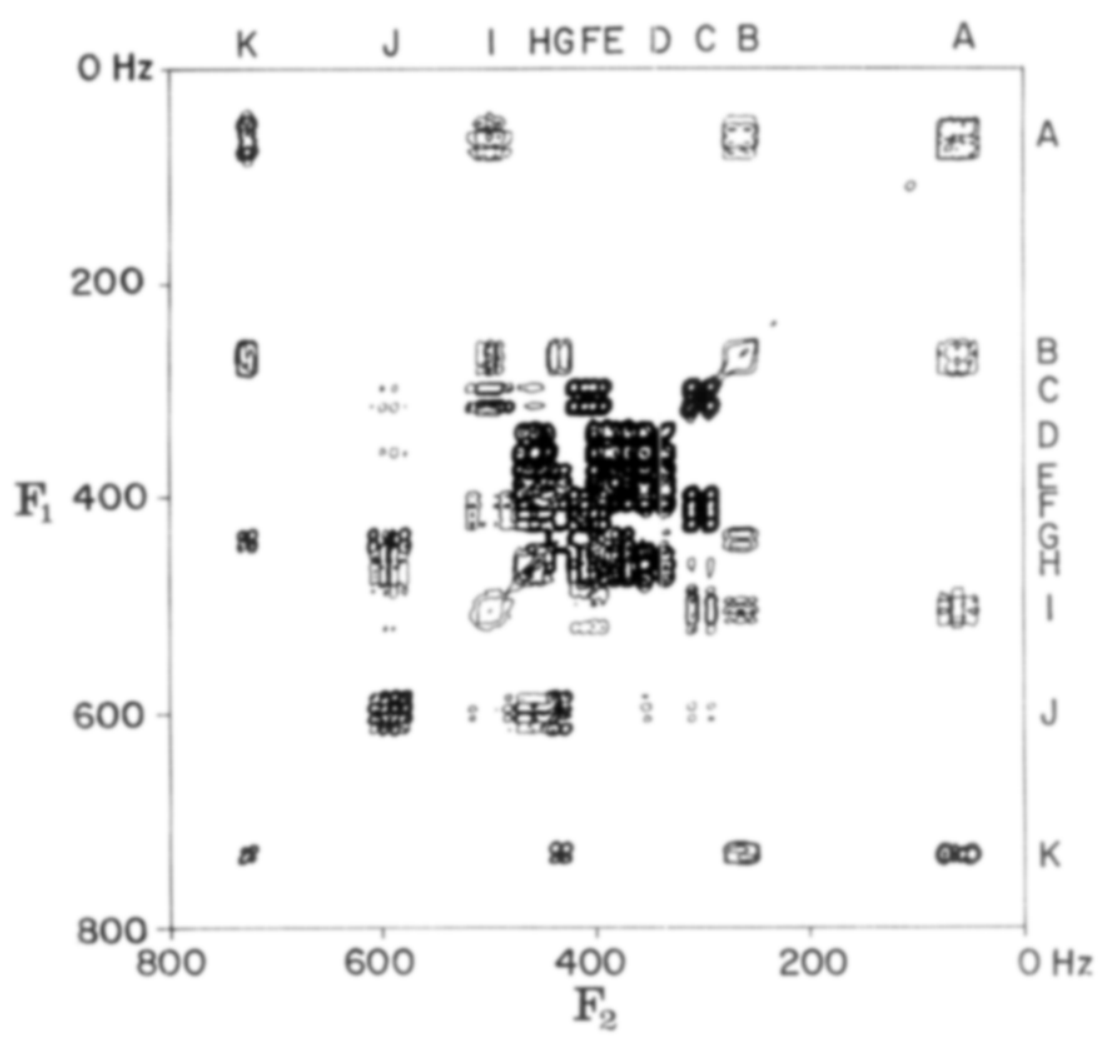

FIG. 5. Contour plot Jeener spectrum of the tricyclodecane derivative. The time-domain signals were multiplied by a shaping function which converts them into "pseudoechoes" with a symmetrical Gaussian envelope. This eliminates dispersion-mode components and leads to a basically circular contour for an isolated line. This spectrum is to be compared with that of Fig. 3. The cross-peaks indicate the spin couplings listed in the left-hand column of Table 1.

For two-dimensional spectra, a Gaussian envelope has advantages. The linewidth is chosen to avoid step-function discontinuities in the time domain, giving a pure Gaussian lineshape in the frequency domain. The same function is used in both $t_{1}$ and $t_{2}$, so that the two-dimensional spectrum has circular intensity contours. Figure 5 shows a Jeener spectrum where the time-domain data have been processed in this manner (see also Table 1).

Apart from enhancing the relative intensity of the cross-peaks, the pseudoecho method has the important property that it can be "tailored" to favor coupling constants of a given magnitude. Optimum transfer of magnetization from one nucleus $A$ to another nucleus $X$ occurs when $\sin \left(\pi J_{\mathrm{Ax}} t_{1}\right) \exp \left(-t_{1} / T_{2 \mathrm{~A}}\right)$ is a maximum, while optimum detection of the transferred magnetization occurs when $\sin \left(\pi J_{\mathrm{Ax}} t_{2}\right) \exp \left(-t_{2} / T_{2 \mathrm{X}}\right)$ is a maximum. (In these expressions, the spin-spin relaxation times $T_{2 \mathrm{~A}}$ and $T_{2 \mathrm{x}}$ have been used rather than the instrumental decay constant $T_{2}^{*}$ for reasons discussed in the following section.) This matching of the shaping function to a particular order of magnitude of coupling constant is very useful for emphasizing long-range coupling constants. If they are so weak that 
TABLE 1

Couplings Observed between Protons in THE. Tricyclodecane Derivative SKETCHED IN FIG. 2

\begin{tabular}{lc}
\hline Normal couplings & Very weak couplings \\
\hline AB, AK, AI & AC, AJ \\
BA, BG, BI, BK & BJ \\
CF, CH, CI, CJ & CA \\
DE, DH, DJ & DG \\
ED, EG, EH & \\
FC, FH, FI & FK \\
GB, GE, GJ, GK & GD \\
HC, HD, HE, HF, HJ & \\
IA, IB, IC, IF, IJ & IK \\
JC, JD, JG, JH, JI & JA, JB, JK \\
KA, KB, KG & KF, KI, KJ \\
\hline
\end{tabular}

$\pi J_{\mathrm{AX}} \ll\left(T_{2 \mathrm{~A}}\right)^{-1},\left(T_{2 \mathrm{X}}\right)^{-1}$ then the optimum choice for the centers of the pseudoechoes is $t_{1}=T_{2 \mathrm{~A}}, t_{2}=T_{2 \mathrm{X}}$. This permits the observation of cross-peaks resulting from spin coupling which is so weak as to be unresolved in the conventional spectrum.

Normally the observation of cross-peaks resulting from very weak couplings would require the storage of a very large data matrix because of the requirement for very fine digitization. This requirement can be relaxed with pseudoecho shaping of the time domain signals by the introduction of a significant "dead time" $\Delta \mathrm{sec}$ between $t_{1}=0$ or $t_{2}=0$ and the beginning of the corresponding pseudoechoes. No data are acquired during the interval $\Delta$. In this way long-range couplings may be investigated without increasing the data storage requirements. In general it is convenient to record the Jeener spectrum under two or more sets of conditions in order to discriminate between cross-peaks from large couplings and those from weak couplings. When the conditions are optimized for weak long-range couplings, then the cross-peaks arising from large couplings can be weak or even absent if there is an integral number of beats in the interval $T / 2$.

The experiment is performed with the same increments and time ranges for $t_{1}$ and $t_{2}$ and the same shaping functions, with the result that the two-dimensional spectrum is exactly symmetrical with respect to $F_{1}$ and $F_{2}$ unless there are spurious responses. This symmetry can be a useful aid in resolving ambiguities, since if a cross-peak is "real" it must have a "twin" symmetrically placed with respect to the principal diagonal.

\section{Coherence Transfer Echoes}

The transient signal after the first radiofrequency pulse, although not normally observed in the spectrometer, decays as a function of $t_{1}$ through natural spin-spin relaxation $\left(T_{2}\right)$ and the effects of spatial inhomogeneity of the magnetic field $\left(T_{2}^{*}\right)$. The same is true of the detection period, except that, if the coherence transfer echo (24) is detected, field inhomogeneity effects are refocused to a good 
approximation. Furthermore, since magnetization transfer takes place within a given molecule, the macroscopic distribution of $B_{0}$ fields has no effect on the process, and the only decay which influences the amplitude of the transferred signal is that resulting from spin-spin relaxation. This produces the surprising result that the amplitude of cross-peaks is dependent on $T_{2}$ but essentially independent of $T_{2}^{*}$. Aue et al. (2) commented on this refocusing effect in the Jeener experiment, which gives rise to very narrow line profiles measured at right angles to the principal axis. There is a close analogy with the spin-tickling double-resonance experiment (25), where the intramolecular nature of the coupling allows $B_{0}$ inhomogeneity effects to be compensated.

This was why, in the section above, the pseudoechoes were centered at times $t_{1}=T_{2}$ and $t_{2}=T_{2}$ rather than $t_{1}=T_{2}^{*}$ and $t_{2}=T_{2}^{*}$, in order to optimize the magnetization transferred through weak long-range couplings. It has been demonstrated that even very weak couplings, an order of magnitude smaller than the natural linewidth, can give detectable cross-peaks. In some spectrometers the instrumental linewidth might be an order of magnitude broader than the natural width. This raises the possibility of detecting the presence of weak couplings many times too small to appear as splittings in the conventional spectrum. However, it would only be possible to get a very rough estimate of the magnitude of such a coupling from the Jeener experiment.

The selection of the coherence transfer echo is achieved by cycling the phase of the second radiofrequency pulse and the reference phase of the receiver in $90^{\circ}$ steps, picking out the component of magnetization which precesses in opposite senses during $t_{1}$ and $t_{2}$ while rejecting the other rotating component, sometimes called the "antiecho" (26).

With a mixing pulse of less than $90^{\circ}$, detection of the coherence transfer echo emphasizes cross-peaks at the expense of diagonal peaks. This is important when the flip angle of the mixing pulse is kept small in order to restrict transfer to directly connected transitions, (see following section), because then a larger proportion of the coherence remains associated with the original nucleus, giving rise to relatively strong diagonal peaks. Discrimination against diagonal peaks is particularly important when there are true cross-peaks near the principal diagonal, as in spin systems with strong coupling $(J \sim \delta)$. An example is provided by the cross-peaks of resonances $\mathrm{D}$ and $\mathrm{E}$ in the spectrum of Fig. 5, where the nuclei are strongly coupled.

\section{Flip Angle Effects}

When the second pulse of the sequence (the "mixing" pulse) is $90^{\circ}$ then magnetization is transferred to all other coupled spins, but it has been shown theoretically (2) that if the mixing pulse has a small flip angle then magnetization is transferred predominantly to connected transitions, those which share a common energy level. This considerably simplifies the resulting two-dimensional spectrum. Furthermore, the transferred magnetization is distributed over a smaller number of cross-peaks and sensitivity can be improved, provided that the flip angle is not too small. In practice a $45^{\circ}$ pulse is a good compromise.

This flip angle effect can be visualized by considering the mixing pulse to be 


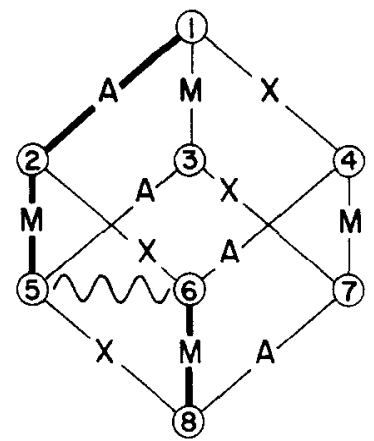

FIG. 6. The energy-level diagram appropriate to the AMX system. An argument based on the concept of pulse cascades has been used to calculate the relative efficiencies of magnetization transfer from $A(12)$ to $M(25)$ and to $M(68)$. The latter transfer involves the forbidden zero-quantum transition (56).

decomposed into a cascade (27) of selective pulses, each affecting only a single transition. Consider the three-spin AMX system with the energy level diagram shown in Fig. 6 . The initial $90^{\circ}$ pulse is not decomposed into a cascade, but simply creates precessing transverse magnetization in all the allowed transitions. The mixing pulse of flip angle $\alpha$ may be represented as a cascade of 12 selective pulses:

$$
\begin{aligned}
& \alpha(12) \alpha(46) \alpha(35) \alpha(78) \\
& \alpha(25) \alpha(13) \alpha(47) \alpha(68) \\
& \alpha(26) \alpha(58) \alpha(14) \alpha(37)
\end{aligned}
$$

Note that the pulses affecting the A spins must all be grouped together, and similarly the pulses affecting the $\mathbf{M}$ spins form a separate group. However, the order of the three groups and the order within any group can be permuted.

Attention may be focused on the fate of one typical transverse magnetization, $A_{X Y}(12)$, and although this precesses as a function $t_{1}$, its amplitude $R_{0}$ is all that needs to be considered here. Consider, first of all, magnetization transfer to a typical connected transition $M_{X Y}(25)$. Not all the elements in the pulse cascade are involved, and it is sufficient to calculate the effect of the cascade:

$$
\alpha(12) \alpha(25) \alpha(26) \alpha(58) .
$$

The first element, $\alpha(12)$, converts transverse magnetization $A_{X Y}(12)$ into longitudinal magnetization $A_{Z}(12)$ of amplitude $R_{0} \sin \alpha$. The second element, $\alpha(25)$, converts one-half of this into transverse magnetization $M_{X Y}(25)$ of amplitude (1/2) $R_{0} \sin ^{2} \alpha$. The remaining two pulses withdraw magnetization from $M_{X Y}(25)$ (creating multiple-quantum coherence) leaving an amplitude (1/2) $R_{0} \sin ^{2} \alpha$ $\cos ^{2}(\alpha / 2)$.

A different sequence of events governs the transfer to a nonconnected transition, $M_{X Y}(68)$, although the same four elements of the pulse cascade are applicable. Transverse magnetization $A_{X Y}(12)$ is converted into $M_{X Y}(25)$ by the first two pulses with amplitude (1/2) $R_{0} \sin ^{2} \alpha$. But the third element, $\alpha(26)$, creates zero-quantum coherence ZQT(56) with an amplitude (1/2) $R_{0} \sin ^{2} \alpha \sin (\alpha / 2)$ and the last element, $\alpha(58)$, reconverts this into observable transverse magnetization $M_{X Y}(68)$ with an 
amplitude (1/2) $R_{0} \sin ^{2} \alpha \sin ^{2}(\alpha / 2)$. A reversal of the sense of the last two elements of the cascade would have given the same result but by way of the double-quantum coherence DQT(28). It is a general rule that transfer to nonconnected transitions involves double- or zero-quantum coherence as an intermediate, whereas directly connected transitions acquire magnetization by conversion of the longitudinal magnetization of a connected transition.

As a result, the ratio of the intensities of connected cross-peaks to those of nonconnected cross-peaks is given by $\cot ^{2}(\alpha / 2)$. Thus for a mixing pulse of $45^{\circ}$ the connected transitions are 5.8 times stronger. This is the setting used in many of the experiments described below when good discrimination between connected and nonconnected transitions is required, as in the determination of relative signs of coupling constants. When sensitivity is an important consideration, a mixing pulse of $60^{\circ}$ delivers higher intensities in the cross-peaks while still retaining a factor of 3 in favor of the connected transitions.

Similar pulse cascade arguments can be used to predict the relative intensities of parallel transitions, where magnetization is transferred between component lines of the same spin multiplet. These lines are situated just off the principal diagonal within a distance of the order of $J$. The general rule is that parallel transitions lose intensity compared with connected transitions as the flip angle is reduced below $90^{\circ}$, but that certain parallel transitions lose intensity faster than others, because they involve more stages of magnetization transfer in succession. In practice it can be very useful to reduce the relative intensity of parallel transitions so as to leave the region close to the principal diagonal clear in order to search for cross-peaks. Figure 7 shows an example of a spectrum where most of the parallel transitions have been reduced below the threshold of the lowestintensity contour. These conclusions, based on the concept of pulse cascades, are consistent with the results derived more formally by Aue et al. (2).

\section{Long-Range Couplings}

The tricyclodecane derivative sketched in Fig. 2 provides a rich field for investigation of long-range proton-proton couplings. The introduction of a relatively long delay $\Delta$ before the beginning of data acquisition enhances the relative intensity of cross-peaks arising from long-range coupling. In practice $\Delta$ was set at $0.3 \mathrm{sec}$. When the coupling constants are much smaller than the natural linewidths, the optimum setting for the peak of the pseudoecho is near points $t_{1}=T_{2}$ and $t_{2}=T_{2}$, where the signal components from long range coupling are maximum. The cross-peak amplitude is then of the order $\sin ^{2}\left(\pi J T_{2}\right) \exp (-2)$ times smaller than a cross-peak from a large spin coupling constant with conventional data acquisition. Sensitivity, however, is still a critical consideration, entailing a compromise setting of $55^{\circ}$ for the flip angle of the mixing pulse.

The intensity contour plot of Fig. 7 was obtained under the conditions described above. It shows six new cross-peaks that were not observed in the spectrum of Fig. 5; they arise from weak long-range couplings. Note that all of these crosspeaks show multiplet structure resulting from large couplings to other protons, the long-range splittings themselves are not of course resolved in this diagram. In addition, some of the cross-peaks resulting from large couplings do not appear 


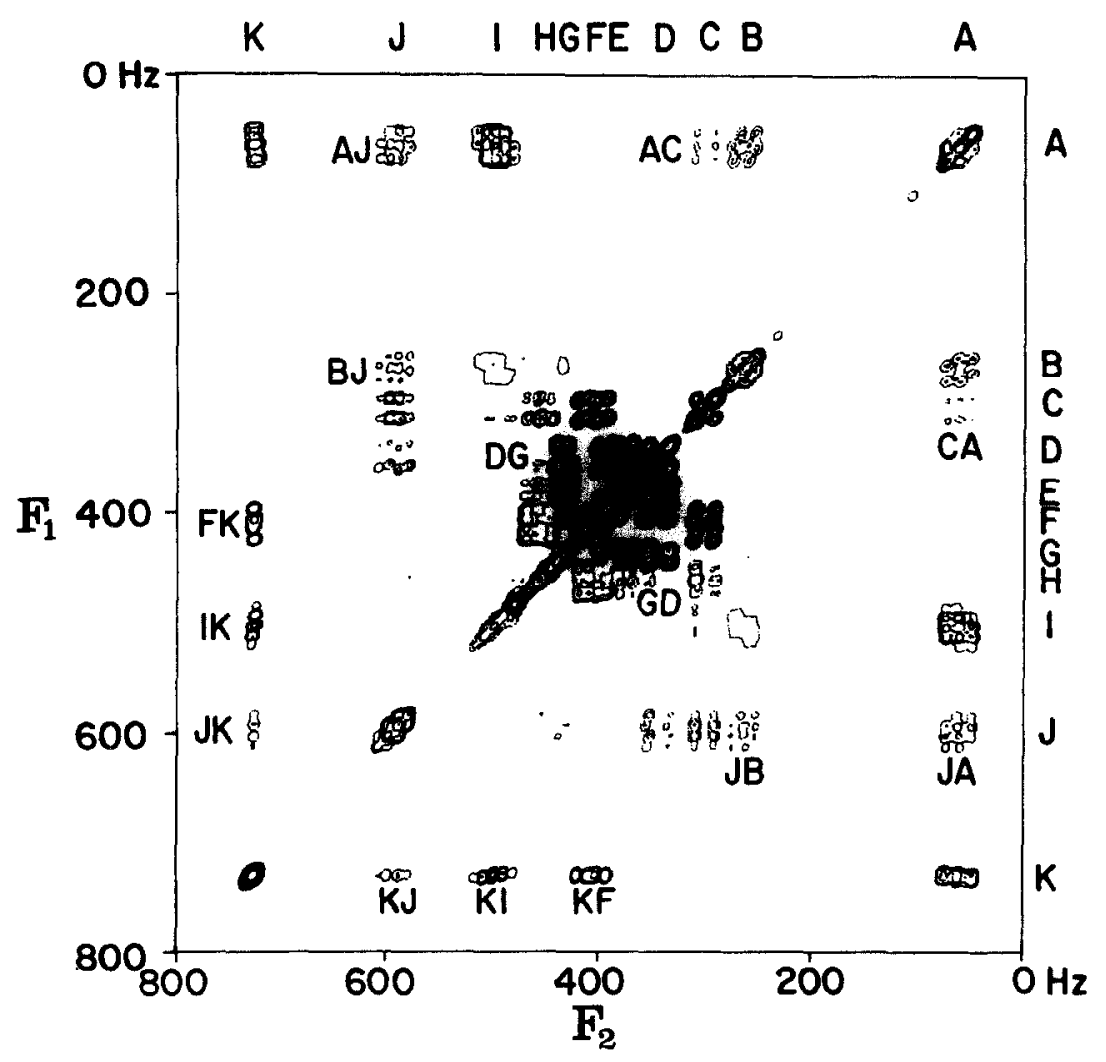

FIG. 7. Jeener spectrum of the tricyclodecane derivative obtained under conditions calculated to emphasize long-range couplings. This involves the use of a reduced flip angle $\left(54.6^{\circ}\right)$ for the mixing pulse, delays $\Delta=0.3 \mathrm{sec}$ before data acquisition in the $t_{1}$ and $t_{2}$ dimensions, and "pseudoecho" shaping of the time-domain signals. The new cross-peaks are indicated and the corresponding couplings are listed in the right-hand column of Table 1.

in Fig. 7 as they have low intensity because of the pseudoecho shaping of the time-domain signals.

An estimate of the approximate magnitudes of these long-range coupling constants would require several experiments with different settings for the peak of the pseudoecho. In a later section, a method of comparing the approximate magnitudes of couplings within the same spectrum is presented. In general the Jeener experiment is quite effective at detecting the presence of weak couplings, but not at estimating their magnitudes.

\section{Relative Signs of Coupling Constants}

There is an interesting consequence of using small flip angles to restrict the transfer of magnetization to connected transitions. In a system of three or more coupled spins, if the appropriate splittings can be resolved, the relative signs of the coupling constants may be ascertained by inspection of the Jeener spectrum. 

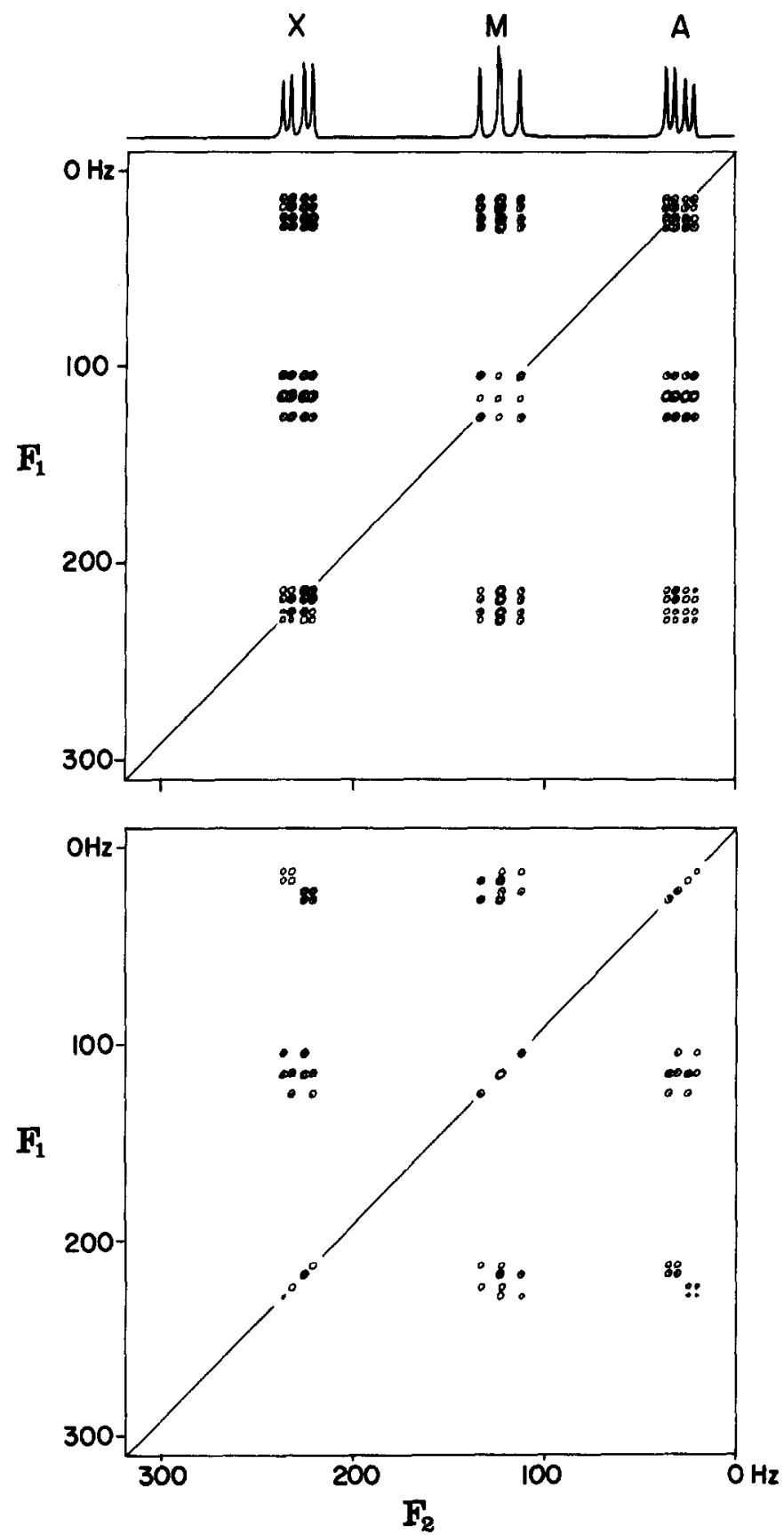

FIG. 8. Contour plots of the Jeener spectra of 2,3-dibromopropionic acid, an AMX spin system. The upper spectrum was obtained with a $90^{\circ}$ mixing pulse and each multiplet contains 16 component lines, grouped in four squares of the kind shown in Fig. 1. The lower spectrum was obtained with a $45^{\circ}$ mixing pulse so that only directly connected transitions are intense enough to show contours. Each cross-peak is now made up of two square patterns, and the sign of the slope of the line joining their centers gives the relative signs of the appropriate coupling constants. 
The basis of the method is the same as that of the selective-decoupling experiments $(28,29)$. Consider the AMX system of 2,3-dibromopropionic acid, where conventional double-resonance and double-quantum experiments have established that the two vicinal couplings have signs opposite to that of the geminal coupling $(30,31)$. When the flip angle of the mixing pulse is small, then AX cross-peaks ir volving a simultaneous flip of the $\mathbf{M}$ nucleus are of vanishingly small intensity; on the other hand, for a flip angle of $90^{\circ}$, all 16 multiplet components are observed with comparable intensity. The Jeener spectrum obtained with a small flip angle thus resembles a selective double-resonance experiment, where $A$ and $X$ are decoupled only in molecules with one of the spin states of the $M$ nucleus, say $M(\alpha)$ but not $M(\beta)$. The local magnetic fields at the sites of the $\mathrm{A}$ and $\mathrm{X}$ nuclei resulting from the couplings to the $\mathrm{M}$ spin must be in the same sense if $J_{\mathrm{AM}} \cdot J_{\mathrm{MX}}$ $>0$. Thus the corresponding displacements of the AX cross-peak are in the same sense in the $F_{1}$ and $F_{2}$ dimensions, resulting in a tilt of the multiplet in the same sense as the principal diagonal. On the other hand, if these couplings have opposite signs, the AX multiplets are tilted in the opposite sense, making an angle greater than $45^{\circ}$ with respect to the principal diagonal. If either $J_{\mathrm{AM}}$ or $J_{\mathrm{MX}}$ is vanishingly small, then the AX multiplet lies vertically on one side of the diagram and horizontally on the other, and no sign determination is possible.

Figure 8 shows the contour diagram for 2,3-dibromopropionic acid, first for a mixing pulse flip angle of $90^{\circ}$, showing each cross-peak made up of 16 components, then for a flip angle of $45^{\circ}$, where each cross-peak has been reduced to $8 \mathrm{com}$ ponents. (In the spectrum obtained at low flip angle, the "parallel" transitionswhere magnetization has been transferred within a single spin multiplet - are absent, leaving only diagonal peaks which fall exactly on the principal diagonal.) Each cross-peak is made up of two groups of four lines which form an exact square (compare Fig. 1). It is the relative orientation of these two squares which determines the relative signs of the coupling constant, each cross-peak relating the signs of two coupling constants. In the case of 2,3-dibromoproprionic acid, $J_{\mathrm{AX}}$ and $J_{\mathrm{MX}}$ have like signs, opposite to the sign of $J_{\mathrm{AM}}$, the geminal coupling.

Once the principle of relative sign determination has been established in a "textbook" case like that of dibromopropionic acid, it is readily applied to more complex spectra simply by noting the tilt of the pattern of lines in a cross-peak. Figure 9

TABLE 2

Relative Signs of Certain Coupling Constants IN THE TRICYCLODECANE DERIVATIVE DETERMINED by Inspection of the JeEner Spectrum in Fig. 9

$\begin{array}{ll}J(\mathrm{AB}) \text { and } J(\mathrm{BK}) & \text { Opposite signs } \\ J(\mathrm{AB}) \text { and } J(\mathrm{BI}) & \text { Opposite signs } \\ J(\mathrm{AI}) \text { and } J(\mathrm{BI}) & \text { Like signs } \\ J(\mathrm{AK}) \text { and } J(\mathrm{AB}) & \text { Opposite signs } \\ J(\mathrm{AB}) \text { and } J(\mathrm{AI}) & \text { Opposite signs } \\ J(\mathrm{CF}) \text { and } J(\mathrm{FI}) & \text { Opposite signs } \\ J(\mathrm{DH}) \text { and } J(\mathrm{EH}) & \text { Like signs }\end{array}$




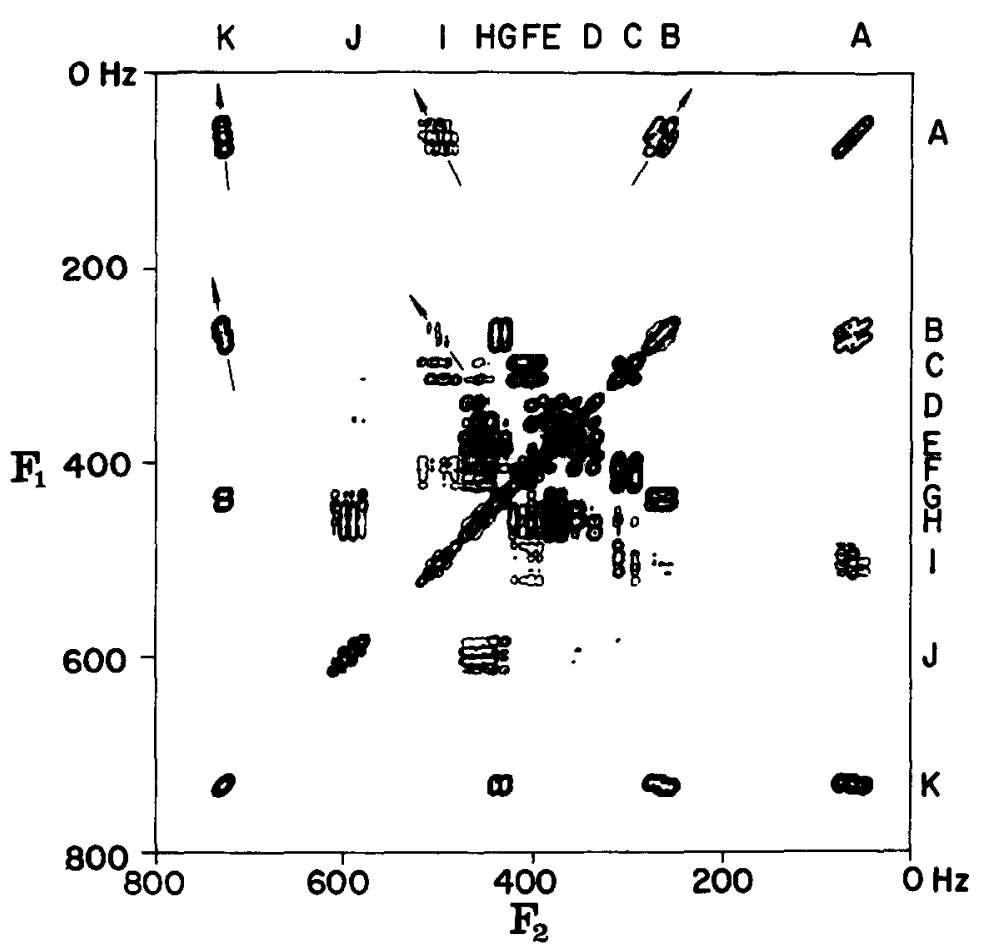

FIG. 9. Jeener spectrum of the tricyclodecane derivative with the conditions adjusted to determine the relative signs of certain coupling constants. The mixing pulse was reduced to $45^{\circ}$ and a "pseudoecho" shaping function used in both time dimensions, but with no dead time $(\Delta=0)$. The arrows indicate the approximate "tilt" of the cross-peaks. The relative signs determined from this spectrum are set out in Table 2.

shows a contour plot of the spectrum of the tricyclodecane derivative obtained with a mixing pulse of flip angle $45^{\circ}$. The tilt of several cross-peaks is indicated by the arrows. Arrows with a positive slope indicate like signs, those with a negative slope opposite signs; a vertical or horizontal slope means that one of the two couplings involved is vanishingly small. (See Table 2.)

The simplicity of the Jeener spectrum obtained with small flip angles for the mixing pulse stems from the fact that each cross-peak is basically from an $\mathrm{AX}$ or $\mathrm{AB}$ spin system; all other splittings are "passive" and simply create several independent $\mathrm{AX}$ or $\mathrm{AB}$ subspectra. In a more general case, groups of two or three spins could be equivalent, leading to $\mathrm{A}_{2} \mathrm{X}, \mathrm{AA}^{\prime} \mathrm{X}$ spectra, for example.

\section{Broadband Decoupling in the $F_{1}$ Dimension}

Throughout the interval between radiofrequency pulses nuclear magnetization vectors corresponding to the components of a given spin multiplet diverge continuously at a rate determined by $J$. Suppose that this interval were to be fixed at a value $t_{\mathrm{d}}$ seconds, and a $180^{\circ}$ refocusing pulse introduced after a variable delay $t_{1} / 2$ after the initial $90^{\circ}$ pulse. Then, although the amplitude of the transferred 


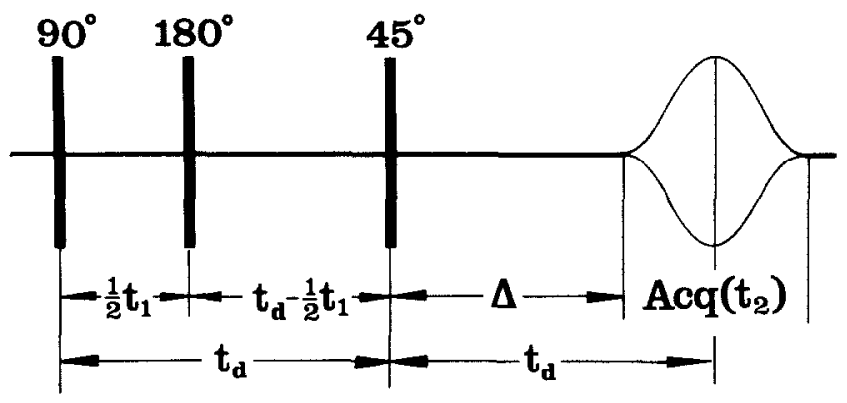

FIG. 10. The pulse sequence used to obtain Jeener spectra that are decoupled in the $F_{1}$ dimension. The delay $t_{\mathrm{d}}$ is fixed, and only $t_{1}$ and $t_{2}$ are varied. A "pseudoecho" shaping function is indicated in the $t_{2}$ dimension. A $45^{\circ}$ mixing pulse is used in order to limit the responses to directly connected transitions.

magnetization would depend on $J t_{\mathrm{d}}$, there would be no $J$ modulation of the signal as $t_{1}$ was varied. On the other hand, the effect of the chemical shift would change, going from a maximum at $t_{1}=0$ to zero when $t_{1}=t_{\mathrm{d}} / 2$ (exact refocusing) and back to a maximum again at $t_{1}=t_{\mathrm{d}}$.

This is the basis of a method of broadband decoupling in the $F_{1}$ dimension (3). It relies on the assumption that the coupling is first order, otherwise spurious responses are excited; in an $A B$ spin system, for example, there is a spurious response at the mean chemical shift frequency (32). By collapsing all spin multiplet structure in the $F_{1}$ dimension onto the appropriate chemical shift frequency, this greatly simplifies the Jeener spectrum, while at the same time concentrating the intensity of the multiplet components. The only information that is lost in this process is that discussed in the previous section-the relative signs of the coupling constants.

A pulse sequence and acquisition scheme (33) is set out in Fig. 10. In this application it is important to employ a mixing pulse of small flip angle $\left(45^{\circ}\right)$ to avoid mutual cancellation of antiphase components within a given cross-peak. The usual phase cycling of the $45^{\circ}$ pulse and the receiver reference phase ensures that the coherence transfer echo is detected. In addition, the $180^{\circ}$ pulse is carefully calibrated and phase alternated along the $\pm Y$ axes of the rotating frame in order to avoid spurious responses from pulse imperfections (18). In this application it is advantageous to increase the digitization along the $F_{1}$ axis in order to take advantage of the improvement in resolution. Figure 11 shows the effect of this broadband decoupling scheme on the Jeener spectrum of the tricyclodecane derivative.

\section{Projections and Cross Sections}

The technique provides an attractive alternative to the established method of obtaining decoupled proton spectra by $45^{\circ}$ projection of two-dimensional $J$ spectra (3). The Jeener spectrum with $F_{1}$ decoupling is simply projected onto the $F_{1}$ axis. Figure 11 shows such a projection together with an indication of the spurious lines 


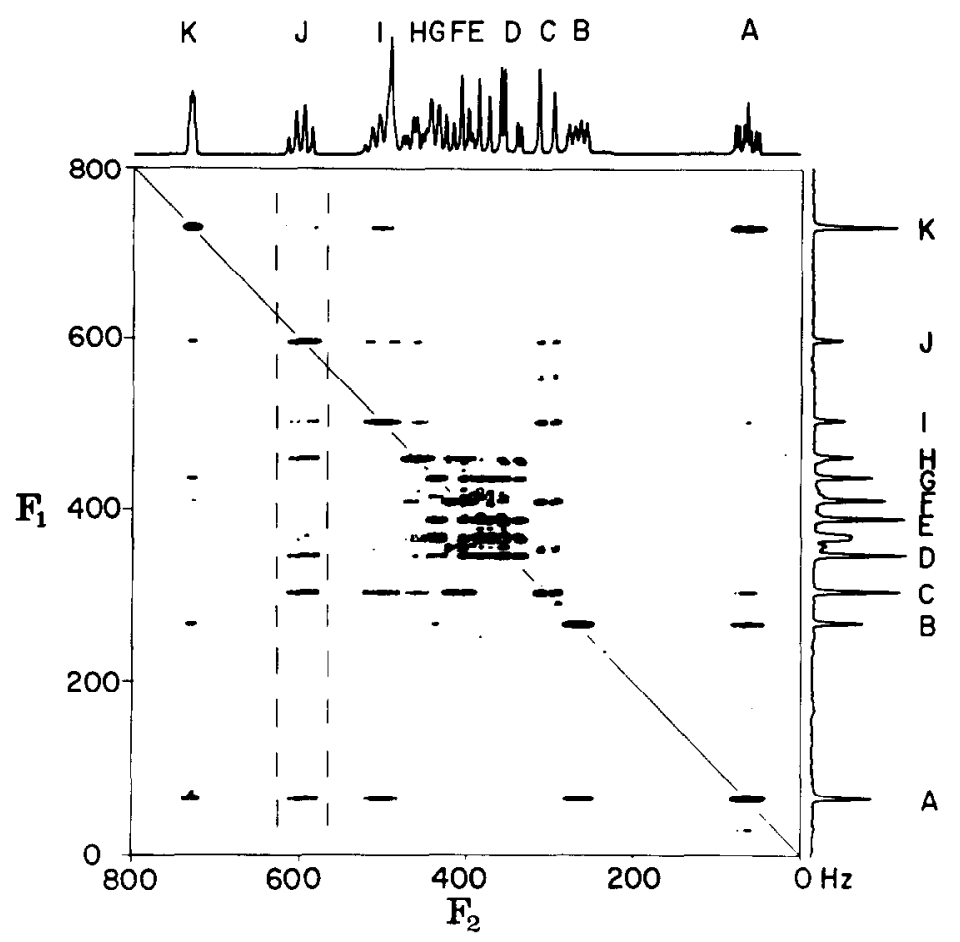

FIG. 11. Jeener spectrum of the tricyclodecane derivative showing broadband decoupling in the $F_{1}$ dimension through the use of the pulse sequence shown in Fig. 10. The spectrum running along the top of the diagram is the fully coupled spectrum; that shown in the right margin is the projection of the two-dimensional spectrum. In this mode the principal diagonal is reversed. A band of signals associated with proton $\mathrm{J}$ has been picked out of the data matrix and projected onto the $F_{1}$ axis in order to obtain the spectrum shown in Fig. 12.

arising from strong coupling effects. Operation with a redefined evolution period $t_{1}$ has resulted in reversing the sense of the principal diagonal in this spectrum.

A cross section through this Jeener spectrum at an appropriate point provides a clear indication of the pattern of couplings to a given proton. For example, if it is required to know which protons are coupled to proton $\mathrm{J}$, a vertical section is taken so as to cut the principal diagonal at the chemical shift of $\mathrm{J}$. In practice it is rather better to extract a vertical band of frequencies from the two-dimensional matrix, as indicated by the dashed lines in Fig. 11; the band is then projected horizontally onto the $F_{1}$ axis. This has the effect of summing all the individual component lines in a given cross-peak. The result is a one-dimensional spectrum (Fig. 12) which indicates the cross-peaks associated with proton J, each cross-peak appearing now as a single line. Not only does this show that proton $\mathrm{J}$ is coupled to A, B, C, D, H, I, and K, but it also gives an approximate estimate of the relative magnitudes of the long-range couplings, for the acquisition parameters were optimized for couplings of the order of the natural linewidth. Large coupling constants give anomalous intensities under these conditions, for example, the crosspeak resulting from coupling between $J$ and $G$ is absent in this trace. On the other 


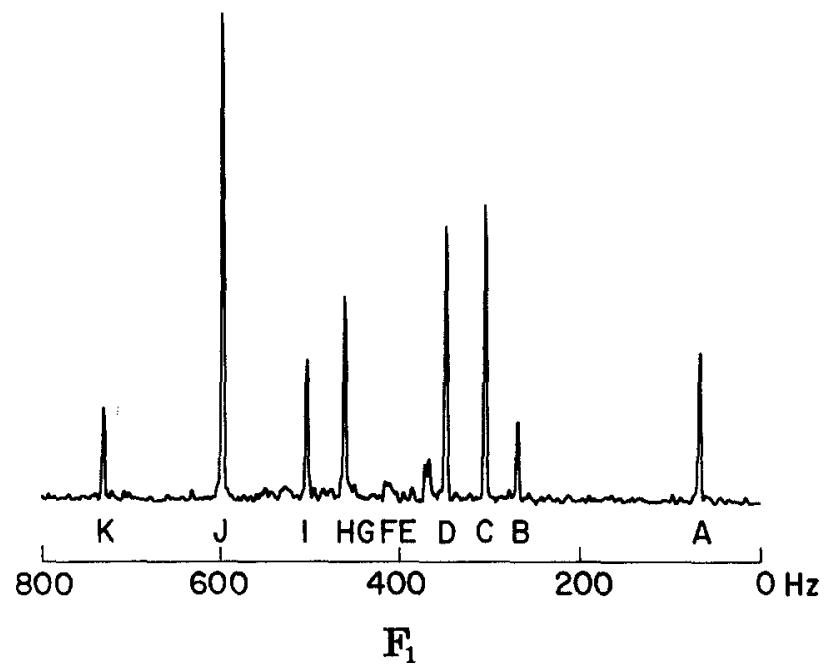

FIG. 12. The projection onto the $F_{1}$ axis of a narrow band of signals extracted from the data matrix of Fig. 11, representing all the cross-peaks associated with proton $\mathrm{J}$. This indicates that $\mathrm{J}$ is coupled to protons A, B, C, D, H, I, and K, and some indication of the relative strengths of these couplings can be obtained provided that they are weak. An artifact appears between the chemical shift frequencies of protons $\mathrm{D}$ and $\mathrm{E}$ because these protons are strongly coupled.

hand, a weak spurious response is observed at the mean chemical shift of protons $\mathrm{D}$ and $\mathrm{E}$, attributable to strong coupling effects.

The pattern of couplings in the tricyclodecane derivative is set out in Table 1. The column "normal couplings" was obtained under acquisition conditions adjusted for relatively strong couplings, with no dead time $\Delta$ before acquisition, using mainly the information from Fig. 5. The column "very weak couplings" was obtained mainly from Fig. 7, where the conditions had been suitably optimized for couplings of the same order as the natural linewidth. In several cases the two sets of results confirmed each other; in other cases they were complementary.

\section{DISCUSSION}

The simple two-dimensional experiment of Jeener (1), although relatively neglected in the past, proves itself well suited to the task of analyzing complex networks of proton-proton spin couplings. In practice it is necessary to modify the operating conditions in various ways in order to simplify the two-dimensional spectra and their interpretation. Reshaping of the two-dimensional responses turns out to be important, and several techniques are used to suppress axial peaks and to reduce the relative intensities of diagonal peaks, in order that attention can be focused on the cross-peaks which carry the coupling information. Intensity contour plots have proved to be the most convenient method of displaying Jeener spectra.

The present work emphasizes the advantage to be gained by operating with a reduced flip angle for the mixing pulse-typically $45^{\circ}$ rather than $90^{\circ}$. It was recog- 
nized at an early stage (2) that this restricts magnetization transfer predominantly to directly connected transitions, but this fact does not seem to have been exploited in chemical applications. In multispin systems there is an interesting side effect of this property. Relative signs of coupling constants are obtained simply by inspection of the "tilt" of two-dimensional spin multiplets, a method closely analogous to the selective decoupling technique $(28,29)$.

Weak long-range couplings, normally hidden within the instrumental linewidth, can be detected unequivocally if suitable changes are made in the method of acquiring the two-dimensional data matrix $S\left(t_{1}, t_{2}\right)$. This involves the introduction of a "dead-time" $\Delta$, and the shaping of the data in the time domain in order to produce a "pseudoecho" which has the property that dispersion-mode contributions to the lineshape are suppressed.

Finally, a simple change in the pulse sequence permits "broadband decoupling" in the $F_{1}$ dimension, further simplifying the two-dimensional spectrum. Projection onto the $F_{1}$ axis then gives a proton spectrum with all scalar splittings removed; indeed partial spectra may be extracted showing only the resonances of protons coupled to a chosen proton. In the proton spectroscopy of large molecules, such a simplification might well prove crucial to the analysis.

\section{ACKNOWLEDGMENTS}

This work was made possible by an cquipment grant from the Science Research Council, and a Stipend from the Delft University Fund (A.B). The tricyclodecane derivative was kindly provided by Mr. P. L. Beckwith.

\section{REFERENCES}

1. J. Jeener, Ampere International Summer School, Basko Polje, Yugoslavia, 1971.

2. W. P. Aue, E. Bartholdi, and R. R. Ernst, J. Chem. Phys. 64, 2229 (1976).

3. W. P. Aue, J. Karhan, And R. R. Ernst, J. Chem. Phys. 64, 4226 (1976).

4. E. L. Hahn and D. E. MaXwell, Phys. Rev. 88, 1070 (1952).

5. R. Freeman and H. D. W. Hill, J. Chem. Phys. 54, 301 (1971).

6. A. A. Maudsley and R. R. ERnst, Chem. Phys. Lett. 50, 368 (1977).

7. A. A. Maudsley, L. Müller, and R. R. Ernst, J. Magn. Reson. 28, 463 (1977).

8. G. Bodenhausen and R. Freeman, J. Magn. Reson. 28, 471 (1977).

9. S. Forsén and R. A. Hoffman, J. Chem. Phys. 39, 2892 (1963).

10. J. Jeener, B. H. Meier, P. Bachmann, and R. R. Ernsi, J. Chem. Phys, 71, 4546 (1979).

11. B. H. Meier and R. R. Ernst, J. Am. Chem. Soc. 101, 6441 (1979).

12. H. Hatanaka, T. Terao, and T. Hashi, J. Phys. Soc. Jpn. 39, 835 (1975).

13. A. Wokaun and R. R. ERnSt, Chem. Phys. Lett. 52, 407 (1977).

14. S. Vega and A. Pines, J. Chem. Phys. 66, 5624 (1977).

15. A. Wokaun and R. R. ERnst, Mol. Phys. 36, 317 (1978).

16. K. Nagayama, K. Wüthrich, and R. R. ERnSt, Biochem. Biophys. Res. Commun. 90, 305 (1979).

17. K. Nagayama, A. Kumar, K. Wüthrich, And R. R. Ernst, J. Magn. Reson. 40, 321 (1980).

18. A. Bax, R. Freeman, AND G. A. Morris, J. Magn. Reson. 42, 164 (1981).

19. R. R. ERNST, Chimia 29, 179 (1975).

20. G. Bodenhausen, R. Freeman, R. Niedermeyer, and D. L. Turner, J. Magn. Reson. 26, 133 (1977).

21. I. D. Campbell, C. M. Dobson, R. J. P. Williams, and A. V. Xavier, J. Magn. Reson. 11, 172 (1973).

22. A. Bax, R. Freeman, And G. A. Morris, J. Magn. Reson. 43, 333 (1981). 
23. A. Bax, A. F. Mehlkopf, And J. Smidt, J. Magn. Reson. 35, 373 (1979).

24. A. A. Maudsley, A. Wokaun, and R. R. Ernst, Chem. Phys. Lett. 55, 9 (1978).

25. R. Freeman and W. A. Anderson, J. Chem. Phys. 37, 2053 (1962).

26. R. Freeman and G. A. Morris, Bull. Magn. Reson. 1, 5 (1979).

27. G. Bodenhausen and R. Freeman, J. Magn. Reson. 36, 221 (1979).

28. D. F. Evans and J. P. Maher, Proc. Chem. Soc. London, 208 (1961).

29. R. Freeman and D. M. Whiffen, Mol. Phys. 4, 321 (1961).

30. R. Freeman, K. A. Mclauchlan, J. I. Musher, and K. G. R. Pachler, Mol. Phys. 5, 321 (1962).

31. K. A. Mclauchlan and D. H. Whiffen, Proc, Chem. Soc. London, 144 (1962).

32. G. Bodenhausen, R. Freeman, G. A. Morris, and D. L. Turner, J. Magn. Reson. 31, 75 (1978).

j3. A. BAX, A. F. MEhlKopf, AND J. SMIDT, J. Magn. Reson. 35, 167 (1979). 